Artículo

\title{
Producción de granos e incidencia de nematodos agalleros en frijol común
}

\author{
Yoerlandy Santana-Baños $1 \S$ \\ Sergio Carrodeguas-Díaz ${ }^{1}$ \\ Irisley Aguiar-González ${ }^{2}$ \\ Adrialys Barroso-Aragón ${ }^{3}$ \\ Armando del Busto-Concepción ${ }^{1}$ \\ Randy López-Alfonso ${ }^{4}$
}

${ }^{1}$ Universidad de Pinar del Río. Calle Martí núm. 300, Barrio Segundo Sur, entre 27 de noviembre y González Alcorta, Pinar del Río, Cuba. CP. 20100. (sergioc@upr.edu.cu; armando@upr.edu.cu). ${ }^{2}$ Empresa Agropecuaria 'Augusto César Sandino', Calle $1^{\text {ra. }}$ C núm. 1 entre Callejón y Límite Zona Industrial, Sandino, Pinar del Río, Cuba. CP. 24150. (irisley85@nauta.cu). ${ }^{3}$ Empresa Agroforestal Guanahacabibes. Manuel Lazo, Sandino, Pinar del Río, Cuba. CP. 24150. (desarrollo@afgbibes.pri.minag.cu). ${ }^{4}$ Centro Meteorológico Provincial. Delegación Territorial del Ministerio de Ciencia Tecnología y Medio Ambiente. Pinar del Río, Cuba. CP. 20100. (randy84@ pri.insmet.cu).

$\S^{\S}$ Autora para correspondencia: yoerlandy@upr.edu.cu.

\section{Resumen}

La interacción genotipo ambiente en cultivares de frijol común (Phaseolus vulgaris L.) y su reacción frente a Meloidogyne spp., son aspectos abordados a pesar de la influencia que tienen en los resultados productivos. Se determinó el potencial productivo de cinco cultivares de frijol común, en agroecosistemas de Pinar del Río, Cuba, y la incidencia de una población de Meloidogyne spp. en fase vegetativa del cultivo. Para ello se establecieron experimentos en 'San Juan y Martínez' y 'Sandino', sobre suelos Ferralítico amarillento y Fluvisol, respectivamente. Se emplearon los cultivares comerciales (tratamientos): 'BAT 304' (testigo de producción comercial), 'CUL 156', 'Buenaventura', 'Delicias 364' y 'Chévere'. También se desarrolló un ensayo en condiciones semicontroladas con macetas de $1.5 \mathrm{~kg}$ y sustrato a base de suelo ferralítico amarillento + turba $(70 \%+30 \%)$, con y sin inoculación de Meloidogyne spp. (1.5 $\mathrm{J}_{2}$-huevos $\mathrm{g}^{-1}$ de sustrato) en los cultivares referidos. Las diferencias entre cultivares y su interacción con los agroecosistemas evidenciaron una marcada influencia sobre la producción de granos, aunque mejores resultados se obtuvieron con los cultivares 'CUL 156' y 'Buenaventura', pues superó en 25\% el rendimiento del testigo de producción comercial, en el agroecosistema de 'San Juan y Martínez' y 'Sandino', respectivamente. Se confirmó que los cultivares evaluados son hospedantes de Meloidogyne spp., aunque 'Buenaventura' expresó menor índice de agallamiento para el nivel de inóculo empleado, mientras que la relación inversa entre fitomasa y el número de agallas y ootecas en el sistema radical de las plantas, sugirieron un efecto nocivo de estos fitonematodos en la fase vegetativa del cultivo.

Palabras clave: Meloidogyne spp., Phaseolus vulgaris L., agroecosistemas, cultivares.

Recibido: enero de 2021

Aceptado: marzo de 2021 


\section{Introducción}

La producción de granos constituye una prioridad a escala mundial y nacional por su importancia en la alimentación humana y animal. En ello juega un importante papel el cultivo del frijol común (Phaseolus vulgaris L.) debido a su valor nutritivo, amplia distribución y consumo (Calero et al., 2018; Aguilar et al., 2019; Martínez et al., 2019). El 67\% de la producción mundial del cultivo, estimada en 30.4 millones de toneladas, se concentró en ocho países: India, Myanmar, Brasil, Estados Unidos de América, China, Tanzania, México y Uganda, por ese orden, todos con cifras superiores al millón de toneladas (FAO, 2018).

En Cuba se cosecharon más de 147000 hectáreas en 2018, con una producción total de 161.5 miles de toneladas y rendimiento agrícola promedio de $1.09 \mathrm{t} \mathrm{ha}^{-1}$ (ONEI, 2019), aunque todavía no satisfacen las necesidades de la población (Martínez et al., 2017; Hernández-Ochandía et al., 2018). $\mathrm{Si}$ se pretende mejorar los resultados productivos en esta leguminosa, es importante que los productores amplíen su germoplasma con cultivares recomendados por su mayor potencial de rendimiento agrícola.

Para lo cual son necesarios los estudios de regionalización dada la interacción genotipo-ambiente que se produce en ese cultivo (Martínez et al., 2019). Sin embargo, en la agricultura cubana existen más de 30 cultivares comerciales en la producción de frijol común (MINAG, 2018), aunque han sido poco estudiados en condiciones agroclimáticas locales de Pinar del Río.

Otro factor con influencia sobre el cultivo del frijol común en Cuba es la afectación por organismos plagas, particularmente de nematodos parásitos de plantas (Hernández-Ochandía et al., 2016), reconociéndose entre estos a Meloidogyne spp. (Nematodos agalleros), causantes de daños de consideración sobre el desarrollo de las plantas (Hernández-Ochandía et al., 2018a), aunque se descuida con frecuencia, en los cultivares empleados o introducidos en la producción, la actitud hospedante frente a estos fitoparásitos, pues son subestimados por técnicos y productores en este cultivo.

Teniendo en cuenta lo anteriormente expuesto, se planteó como objetivo determinar el potencial productivo de cultivares de frijol común, en agroecosistemas de Pinar del Río, Cuba y la incidencia de una población de Meloidogyne spp., en fase vegetativa del cultivo.

\section{Materiales y métodos}

\section{Descripción de escenarios para los experimentos de campo}

Los ensayos de campo se desarrollaron en el periodo de noviembre 2017 a febrero 2018, en los consejos populares 'Río Seco' ( $22^{\circ} 18^{\prime} 13^{\prime}$ ” latitud norte y $83^{\circ} 47^{\prime}$ '39”' longitud oeste) de 'San Juan y Martínez' y 'Sandino' (SJM) y 'Manuel Lazo' (22 $02^{\circ}$ ' 95” latitud norte y $84^{\circ} 21^{\prime}$ ' 22” longitud oeste) de 'Sandino' (SDN), en Pinar del Río, Cuba. El suelo de las localidades se clasificó como Ferralítico Amarillento $\left(\mathrm{pH}_{(\mathrm{KCl})}=5.1\right.$ y $\left.\mathrm{MO}=1.84 \%\right)$ y Fluvisol $\left(\mathrm{pH}_{(\mathrm{KCl})}=4.71\right.$ y $\left.\mathrm{MO}=1.51 \%\right)$, respectivamente, según la clave de clasificación de suelos de Cuba (Hernández et al., 2015). 
Las condiciones climáticas durante los ensayos en San Juan y Martínez (Figura 1A) y Sandino (Figura 1B), se caracterizaron por temperaturas medias de $22.9^{\circ} \mathrm{C}$ y 23.3 , humedad relativa de $77.9 \%$ y $75.9 \%$ y precipitaciones acumuladas de $150.5 \mathrm{~mm}$ y $142.5 \mathrm{~mm}$, según datos obtenidos en las estaciones meteorológicas número 314 (San Juan y Martínez) y número 313 (Sandino) del centro meteorológico provincial.

(A)

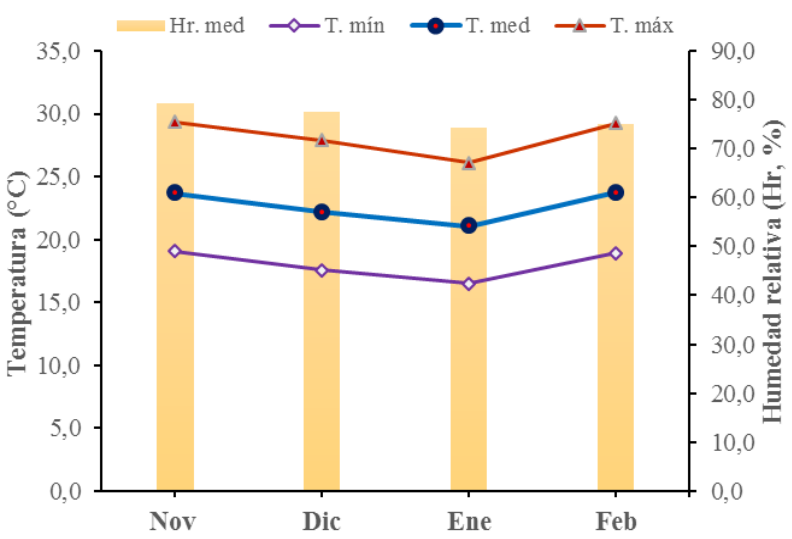

(B)

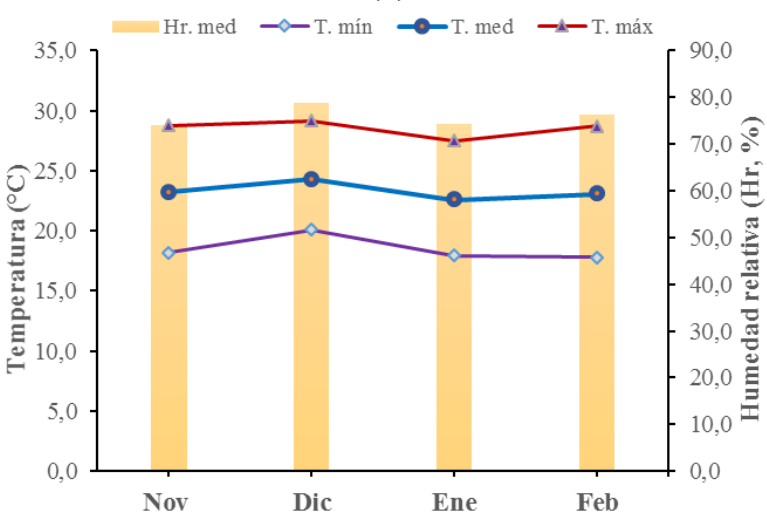

Figura 1. Valores promedios de temperatura (mínima, media, máxima) y humedad relativa en 'San Juan y Martínez' (A); y 'Sandino' (B).

\section{Cultivares empleados y experimentos de campo}

Se emplearon cinco cultivares (tratamientos) de frijol común (Cuadro 1), distribuidos en un diseño de bloques al azar con cuatro réplicas. La superficie cultivada, en cada localidad, fue de $560 \mathrm{~m}^{2}$ con unidades experimentales de $28 \mathrm{~m}^{2}$. Se garantizó uniformidad en el manejo del cultivo en las dos localidades. La siembra se realizó de forma manual, a una distancia de $0.6 \mathrm{~m}$ entre surcos y 0.07 entre plantas.

Cuadro 1. Descripción de los cultivares empleados en los experimentos.

\begin{tabular}{cccc}
\hline Cultivares & Color & Tipo de crecimiento & Rendimiento agrícola potencial (t ha $\left.{ }^{-1}\right)$ \\
\hline 'BAT 304' & Negro & Indeterminado postrado & 2.1 \\
'CUL 156' & Negro & Indeterminado arbustivo & 3.17 \\
'Buenaventura' & Rojo & Indeterminado arbustivo & 2.93 \\
'Delicias 364' & Rojo & Indeterminado arbustivo & 2.8 \\
'Chévere' & Blanco & Indeterminado postrado & 3.1 \\
\hline
\end{tabular}

* = testigo de producción comercial de frijol común en las localidades de estudio (Faure et al., 2013).

Todas las atenciones culturales se realizaron según lo establecido en la guía técnica para el cultivo del frijol en Cuba (Faure et al., 2013). La fertilización se utilizó con la fórmula 12-6-16-3 (N-P-K$\mathrm{Mg}$ ) al momento de la siembra, a una dosis de $0.4 \mathrm{t} \mathrm{ha}^{-1}$, y una aplicación foliar de fitomas E (1.5 $\mathrm{L} \mathrm{ha}^{-1}$ ) previo a la floración. Para el riego se empleó el método superficial por surco. En la protección del cultivo se utilizaron los plaguicidas Celest Top FS 31.2 (tiametoxan $26.2+$ difenoconazol 2.5 + fludioxonilo 2.5), hidróxido de calcio $(\mathrm{CaOH})$, mezcla duple $\mathrm{E} 3.125$ (acefato 3 + cipermetrina 0.125 ) y Kospi SC 13 (imidacloprid 10.0 + bifentrina 3 ). 


\section{Variables evaluadas en los experimentos de campo}

Las evaluaciones del rendimiento y sus componentes se realizaron al momento de la cosecha. Se seleccionaron 10 plantas representativas al azar por réplica en cada cultivar. Las variables analizadas fueron: número de legumbres/planta (u), índice de legumbres llenas (\%) -valorado como proporción de legumbres con más de $50 \%$ de cuajado de la semilla-, número de semillas/legumbre (u), masa de la legumbre (g), masa de semillas/legumbre (g), masa de 100 semillas (g) y rendimiento agrícola $\left(\mathrm{t} \mathrm{ha}^{-1}\right)$.

El cálculo de las variables relacionadas con la masa se empleó de una balanza técnica digital OHUS Adventurer ${ }^{\circledR}$ Pro de precisión $0.01 \mathrm{~g}$. Para determinar el rendimiento, se cosecharon $6 \mathrm{~m}^{2}$ del centro en cada réplica, se trillaron las plantas y se secaron los granos a 14\% de humedad.

\section{Experimento para la interacción cultivares-Meloidogyne spp.}

El experimento se desarrolló entre febrero y marzo de 2018, en condiciones semicontroladas de la Universidad de Pinar del Río, Cuba, con localización en los $22^{\circ} 24^{\prime} 48^{\prime \prime}$ latitud norte y $83^{\circ} 41^{\prime} 16^{\prime}$ " longitud oeste. Las condiciones ambientales se caracterizaron por temperaturas entre los 26 y 36.2 ${ }^{\circ} \mathrm{C}$, con media de $30.2{ }^{\circ} \mathrm{C}$, mientras que la humedad relativa osciló entre 54 y $82 \%$. Para el monitoreo de las variables se empleó una Weather Pocket Station marca LM-8000 DC9V.

Se empleó un diseño completamente al azar con arreglo factorial. Se consideraron dos factores: cultivares ('BAT 304', 'CUL 156', 'Buenaventura', 'Delicias 364' y 'Chévere') y nematodos (con y sin inoculación de Meloidogyne spp.) y cinco replicas por tratamiento. La siembra se realizó en macetas de $1.5 \mathrm{~kg}$ de sustrato, el cual se elaboró a base de suelo ferralítico amarillento (Hernández et al., 2015) + turba $(70 \%+30 \%)$, previamente desinfestado con formol al $4 \%$, con $\mathrm{pH}_{(\mathrm{KCl})}=5.84$ y $\mathrm{MO}=6.67 \%$. El inóculo de Meloidogyne spp., procedente de una población asociada al cultivo del frijol común en el suelo empleado, se obtuvo siguiendo la metodología de Hussey y Barker (1973).

El mismo se aplicó cinco días después de la siembra a razón de $1.5 \mathrm{~J}_{2}$-huevos $\mathrm{g}^{-1}$ de sustrato. A los 35 días después de la germinación, se analizaron las variables morfofisiológicas en las platas de frijol de los cultivares evaluados: longitud del tallo $(\mathrm{cm})$, diámetro del tallo $(\mathrm{mm})$, longitud del sistema radical $(\mathrm{cm})$, número de raíces secundarias y masa fresca total $(\mathrm{g})$. También se cuantificó, en el sistema radical de las plantas, la infestación por Meloidogyne spp., expresada en número de agallas y ootecas por gramo de raíz, y el número de nódulos por cepas nativas de Rhizobium spp. La observación y conteo directo se realizaron con ayuda del estereoscopio Novel $^{\circledR}$.

\section{Análisis estadísticos de los resultados}

Con los datos obtenidos en los experimentos, se procedió a comprobar los supuestos de normalidad y homogeneidad de varianza mediante las pruebas Kolmogorov-Smirnov y Levene, respectivamente. Se aplicó análisis de varianza, en correspondencia con los diseños experimentales, y prueba de Tukey para la comparación de medias, con un nivel de confianza de $95 \%(p \leq 0.05)$. También se realizó análisis de componentes principales para el experimento de interacción cultivares - Meloidogyne spp. Se empleó el software estadístico Minitab 17 para windows. 


\section{Resultados y discusión}

\section{Efecto de la interacción cultivares agroecosistemas sobre el rendimiento de frijol común}

Los resultados arrojaron diferencias altamente significativas para todas las variables analizadas en los dos factores incluidos, con excepción del rendimiento agrícola entre agroecosistemas (Cuadro 2); sin embargo, sólo se encontró interacción significativa para el número de legumbres/planta (L/P), masa de legumbre (ML) y rendimiento agrícola (RA). Ello demuestra la importancia de los estudios de interacción genotipo ambiente en la selección de cultivares para condiciones agroclimáticas locales y constituyen el primer informe sobre la temática para estas localidades de Pinar del Río, Cuba.

Cuadro 2. Análisis de varianza bifactorial (valor F) para los componentes de rendimiento.

\begin{tabular}{cccccccc}
\hline Factores & L/P & ILL & S/L & ML & MS/L & M100S & RA \\
\hline Cultivares & $38.34^{* *}$ & $24.36^{* *}$ & $44.56^{* *}$ & $18.01^{* *}$ & $35.42^{* *}$ & $39.24^{* *}$ & $20.5^{* *}$ \\
Agroecosistemas & $17.73^{* *}$ & $60.1^{* *}$ & $11.59^{* *}$ & $9.58^{* *}$ & $26.53^{* *}$ & $6.53^{* *}$ & $2.04^{\mathrm{ns}}$ \\
Cultivares*agroecosistemas & $6.19^{* *}$ & $1.93^{\mathrm{ns}}$ & $0.54^{\mathrm{ns}}$ & $3.28^{*}$ & $2.35^{\mathrm{ns}}$ & $1.79^{\mathrm{ns}}$ & $9.89^{* *}$ \\
EEM & 0.22 & 1.38 & 0.09 & 0.02 & 0.02 & 0.32 & 0.04 \\
\hline
\end{tabular}

$\mathrm{L} / \mathrm{P}=$ legumbres/planta; ILL= índice de legumbres llenas; $\mathrm{S} / \mathrm{L}=$ semillas/legumbre; $\mathrm{ML}=$ masa de la legumbre; $\mathrm{MS} / \mathrm{L}=$ masa de semillas/legumbre; $\mathrm{M} 100 \mathrm{~S}=$ masa de cien semillas; $\mathrm{RA}=$ rendimiento agrícola; ${ }^{* *},{ }^{*}=$ diferencias significativas para $p \leq 0.01$ y $p \leq 0.05 ;{ }^{\text {ns }}=$ no significativo; EEM= error estándar de la media.

Algunos autores han encontrado diferencias altamente significativas al comparar el rendimiento y sus componentes entre cultivares de frijol común (Izquierdo et al., 2018; Romero et al., 2019), de ahí la importancia de valorar, además, el potencial que expresan en las condiciones agroclimáticas evaluadas. Con relación a los componentes del rendimiento agrícola, se puede apreciar que las diferencias entre los cultivares (Cuadro 3) indican mejores resultados con 'CUL 156' para el número de legumbres/planta, semillas/legumbre, masa de legumbre y masa de semillas/legumbre.

Cuadro 3. Valores promedios de los componentes de rendimiento por cultivar y agroecosistema.

\begin{tabular}{ccccccc}
\hline Factores & L/P $(\mathrm{u})$ & ILL $(\%)$ & S/L $(\mathrm{u})$ & ML $(\mathrm{g})$ & MS/L $(\mathrm{g})$ & $\mathrm{M} 100 \mathrm{~S}(\mathrm{~g})$ \\
\hline & & \multicolumn{7}{c}{ Cultivares } \\
BAT 304' & $8.96 \mathrm{c}$ & $88.72 \mathrm{a}$ & $5.06 \mathrm{~b}$ & $1.07 \mathrm{c}$ & $0.8 \mathrm{c}$ & $16.13 \mathrm{c}$ \\
'CUL 156' & $13.86 \mathrm{a}$ & $65.13 \mathrm{c}$ & $7.56 \mathrm{a}$ & $1.52 \mathrm{a}$ & $1.35 \mathrm{a}$ & $17.79 \mathrm{bc}$ \\
'Buenaventura' & $11.23 \mathrm{~b}$ & $74.32 \mathrm{~b}$ & $4.39 \mathrm{c}$ & $1.34 \mathrm{~b}$ & $1.02 \mathrm{~b}$ & $23.19 \mathrm{a}$ \\
'Delicias 364' & $10.27 \mathrm{~b}$ & $76.04 \mathrm{~b}$ & $4.86 \mathrm{~b}$ & $1.08 \mathrm{c}$ & $0.85 \mathrm{c}$ & $17.54 \mathrm{bc}$ \\
'Chévere' & $8.91 \mathrm{c}$ & $79.21 \mathrm{~b}$ & $4.76 \mathrm{~b} \mathrm{c}$ & $1.29 \mathrm{~b}$ & $0.91 \mathrm{c}$ & $19.09 \mathrm{~b}$ \\
& & Agroecosistemas & & \\
San Juan y Martínez & 12.6 & 61.6 & 5.68 & 1.42 & 1.16 & 20.8 \\
Sandino & 9.28 & 85.99 & 4.69 & 1.15 & 0.84 & 18.18 \\
\hline
\end{tabular}

Letras distintas en una misma columna indican diferencias significativas $(p \leq 0.05) . \mathrm{L} / \mathrm{P}=$ legumbres/planta; ILL= índice de legumbres llenas; $\mathrm{S} / \mathrm{L}=$ semillas/legumbre; $\mathrm{ML}=$ masa de la legumbre; $\mathrm{MS} / \mathrm{L}=$ masa de semillas/legumbre; $\mathrm{M} 100 \mathrm{~S}=$ masa de cien semillas. 
Cabe destacar, que un mejor resultado en número de legumbres/planta es clave para la producción del cultivo debido a su reconocido aporte al rendimiento agrícola (De la Fé et al., 2016); Izquierdo et al., 2018). Un estudio de 15 cultivares de frijol común en la región oriental de Cuba indicó valores entre 13.9 y 23.6 legumbres/planta (Estrada et al., 2016), resultado que corrobora la variabilidad de este componente entre cultivares y agroecosistemas.

El número de semillas/legumbre contrastó con otras investigaciones desarrolladas en Cuba, pues Maqueira et al. (2017) alcanzaron valores inferiores a 4 semillas/legumbre en 'CUL 156', para igual fecha de siembra, y Calero et al. (2018) obtuvieron entre 3 y 4 semillas/legumbre con 'Buenaventura'. El índice de legumbres llenas arrojó valores significativamente superiores en el testigo de producción comercial 'BAT 304', aunque todos superaron $60 \%$.

Valores superiores en este índice sugieren mayor proporción de fotosintatos hacia la producción de semilla (Flores et al., 2018) y a criterio de los autores, presenta una relación directa con el índice de cosecha en el cultivo; sin embargo, la producción de granos no siempre alcanza resultados superiores con cultivares más eficientes, pues en ello influye el potencial de rendimiento agrícola de cada uno, aunque es indispensable para la producción de semillas en este cultivo. La masa seca de 100 semillas superó lo establecido para este componente en los cultivares 'Buenaventura' y 'Chévere', los demás cultivares expresaron más de $75 \%$ de su masa promedio (Faure et al., 2013).

Los mejores resultados entre agroecosistemas para los componentes del rendimiento, con excepción del índice de legumbres llenas, se obtuvieron en 'San Juan y Martínez', con valores que superan entre $14 \%$ y $38 \%$ lo alcanzado en 'Sandino'. Sin embargo, en esta localidad, el índice de legumbres llenas superó en $28.4 \%$ el valor alcanzado en 'San Juan y Martínez', donde se logró un mayor desarrollo vegetativo y como consecuencia de ello, una menor proporción de fotosintatos destinados a las semillas en la fase reproductiva del cultivo.

En el rendimiento agrícola (Figura 2) se pudo constatar que el testigo de producción comercial 'BAT 304' fue superado, significativamente, por los cultivares 'CUL 156' y 'Buenaventura' en los agroecosistemas de 'San Juan y Martínez' y 'Sandino', respectivamente, con incrementos superiores a $25 \%$ en la producción de granos. Se destaca que sólo el cultivar 'Buenaventura' arrojó diferencias significativas entre agroecosistemas, aunque, de manera general, los cultivares negros ('BAT 304' y 'CUL 156') y blanco ('Chévere') alcanzaron medias superiores de rendimiento agrícola en 'San Juan y Martínez' y los rojos ('Buenaventura' y 'Delicias 364') en 'Sandino', independientemente de las diferencias estadísticas.

Se apreció, además, que los cultivares 'BAT 304' (en ambos agroecosistemas) y 'Buenaventura' (en 'Sandino'), superaron 60\% de su rendimiento agrícola potencial (Cuadro 1). Sin embargo, el rendimiento agrícola promedio, en todos los cultivares, superó lo alcanzado por el cultivo del frijol común en Cuba (1.09 t ha ${ }^{-1}$ ), según los datos de la Oficina Nacional de Estadísticas e Información (ONEI, 2019), resultado de gran importancia no sólo en la producción de granos, sino para la selección de escenarios destinados a la producción de semillas de estos cultivares, pues se conoce que, en la mayoría de las zonas productoras de frijol común, los rendimientos potenciales nunca son alcanzados (Domínguez et al., 2016). 


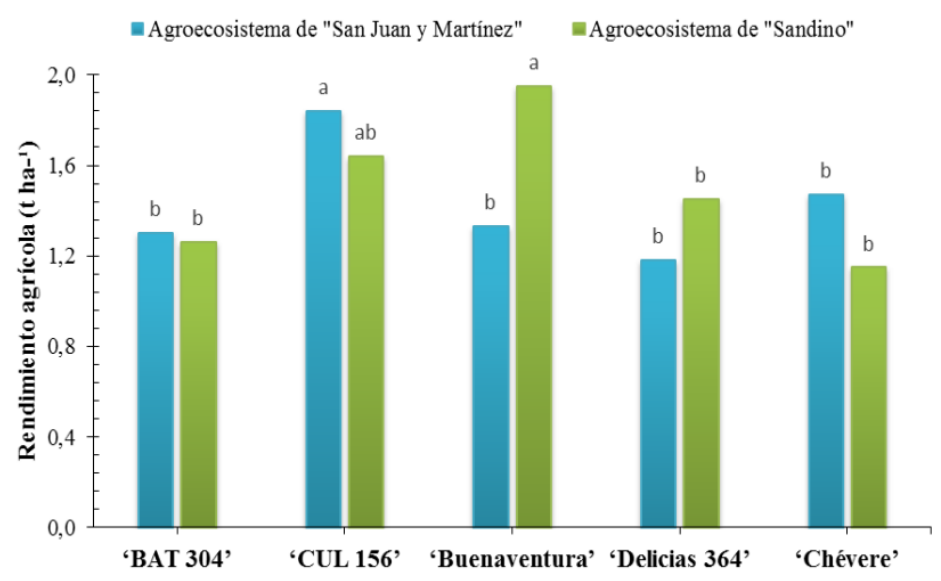

Figura 2. Efecto de la interacción cultivares agroecosistemas sobre el rendimiento agrícola del cultivo. Letras distintas en las barras indican diferencias significativas para $p \leq 0.05$.

Estudios desarrollados en agroecosistemas cubanos, donde se incluyeron los cultivares 'CUL 156', 'Buenaventura' y 'Delicias 364', informan mejores resultados productivos en estos últimos, con valores de rendimiento agrícola superiores a $1.5 \mathrm{t} \mathrm{ha}^{-1}$ (Izquierdo et al., 2018); sin embargo, De la Fé et al. (2016) obtuvieron entre 1 y $3.2 \mathrm{t} \mathrm{ha}^{-1}$ en 14 cultivares de reciente introducción en la producción, mientras que Martínez et al. (2015) superaron las $2 \mathrm{t} \mathrm{ha{ } ^ { - 1 }}$ con el cultivar 'Chévere'.

\section{Efecto de la población de Meloidogyne spp. sobre los cultivares de frijol común}

Se destaca que los cinco cultivares empleados son hospedantes de Meloidogyne spp. (Figura 3) y permiten su reproducción, pues se observó agallamiento y presencia de ootecas externas en el sistema radical, que confirma el criterio de varios autores que consideran a estos fitonematodos como plagas en frijol común (Hernández-Ochandía et al., 2016; Al-Hazmi et al., 2017).

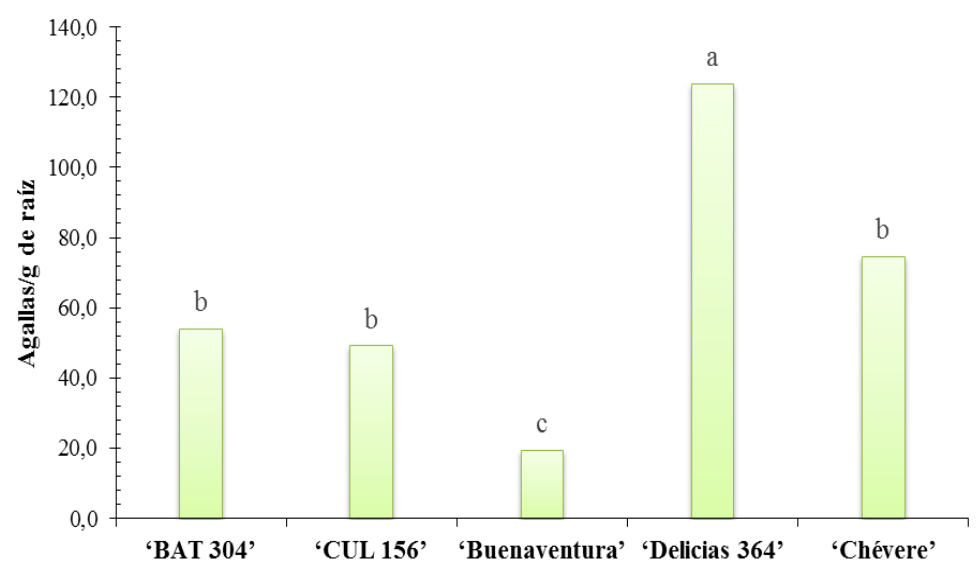

Figura 3. Capacidad de agallamiento de Meloidogyne spp., en los cultivares de frijol común. Letras distintas en las barras indican diferencias significativas para $p \leq 0.05$.

El mejor resultado se obtuvo en el cultivar 'Buenaventura', con agallamiento inferior al 50\% de lo expresado por otros cultivares evaluados, mientras que la mejor actitud hospedante se apreció en el cultivar 'Delicias 364', superó dos veces la infestación de 'BAT 304'. Lo anterior, debe 
profundizarse en estudios de resistencia/susceptibilidad para definir cultivares de referencia en estudios de interacción frijol común - nematodos (Meloidogyne spp.). No obstante, lo expuesto constituye referente científico para este cultivo en las condiciones de Pinar del Río, Cuba.

El análisis multivalente para la interacción cultivares Meloidogyns spp. Sobre el desarrollo vegetativo evidenció dos nuevos componentes principales (CP) extraídas que explicaron, en su conjunto $86.9 \%$ de la varianza de los resultados, con coeficientes de extracción >0.65 (Cuadro 4).

Cuadro 4. Matriz de correlación entre las componentes principales extraídas y las variables originales para la interacción cultivares-Meloidogyne spp.

\begin{tabular}{|c|c|c|c|}
\hline \multirow{2}{*}{ Variables originales } & \multicolumn{2}{|c|}{ Componentes principales } & \multirow{2}{*}{ Extracción } \\
\hline & $\mathrm{CP} 1$ & $\mathrm{CP} 2$ & \\
\hline Diámetro del tallo & 0.188 & 0.902 & 0.914 \\
\hline Masa fresca total & 0.905 & 0.309 & 0.849 \\
\hline Masa seca total & 0.965 & -0.089 & 0.938 \\
\hline Agallas g ${ }^{-1}$ de raíz & -0.693 & 0.387 & 0.951 \\
\hline Ootecas g ${ }^{-1}$ de raíz & -0.917 & 0.234 & 0.897 \\
\hline Nódulos de Rhizobium $\mathrm{g}^{-1}$ de raíz & 0.426 & 0.623 & 0.665 \\
\hline Varianza explicada & 52.8 & 34.12 & 86.92 \\
\hline
\end{tabular}

Método de extracción= análisis de componentes principales.

La primera componente principal (CP1) estuvo determinada por la masa fresca y seca totales y la infestación de Meloidogyne spp. (agallas y ootecas por gramo de raíz), evidenciándose que la fitomasa de las plantas de frijol aumentó con la reducción del número de ootecas y agallas, lo que sugiere un efecto nocivo de los fitonemtodos sobre los cultivares de frijol común, el cual se acentúa con la completa reproducción de la hembra, pues se obtuvo una alta correlación (>0.9 en módulo) negativa entre el número de ootecas por gramo de raíz y las fitomasas fresca y seca, resultado que corrobora lo expresado por varios autores sobre la acción parasítica de Meloidogyne spp. En frijol común (Al-Hazmi et al., 2017; Hernández-Ochandía et al., 2018a).

Este resultado sugiere profundizar en la tolerancia de los cultivares frente a diferentes niveles de inóculos de Meloidogyne spp., tanto en fase vegetativa como reproductiva, para definir su impacto sobre crecimiento y rendimiento de este. La segunda componente principal (CP2) se relacionó con las variables originales diámetro del tallo y número de nódulos de rizobios nativos, las cuales expresaron una correlación positiva, derivada del efecto de la fijación biológica de nitrógeno resultante de la simbiosis frijol común-Rhizobium.

\section{Conclusiones}

Las diferencias entre cultivares y su interacción con los agroecosistemas evidenciaron una marcada influencia sobre la producción de granos, aunque los mejores resultados se obtuvieron con los cultivares 'CUL 156' y 'Buenaventura', pues superaron en $25 \%$ el rendimiento agrícola del testigo de producción comercial, en el agroecosistema de 'San Juan y Martínez' y 'Sandino', respectivamente. 
Todos los cultivares evaluados fueron hospedantes de Meloidogyne spp., aunque se alcanzó menor índice de agallamiento en 'Buenaventura' y se constató que el incremento del número de agallas y ootecas, en el sistema radical de las plantas, redujo la fitomasa en la fase vegetativa del cultivo.

\section{Literatura citada}

Aguilar, G.; Vázquez, E. G.; Castro, R.; Cruz, E. y Jarquín, R. 2019. Germinación de cultivares de frijol con características físicas contrastantes bajo condiciones de estrés osmótico. Rev. Mex. Cienc. Agríc. 10(2):239-251.

Al-Hazmi, A. S.; Dawabah, A. A. M.; Al-Nadhari, S. N. and Al-Yahya, F. A. 2017. Comparative efficacy of different approaches to managing Meloidogyne incognita on green bean. Saudi J. Biol. Sci. 24(1):149-154.

Calero, A.; Castillo, Y.; Quintero, E.; Pérez, Y. y Olivera, D. 2018. Efecto de cuatro densidades de siembra en el rendimiento agrícola del frijol común (Phaseolus vulgaris L.). Rev. Fac. Cienc. 7(1):88-100.

De La-Fé, C. F.; Lamz, A.; Cárdenas, R. M. y Hernández, J. 2016. Respuesta agronómica de cultivares de frijol común (Phaseolus vulgaris L.) de reciente introducción en Cuba. Cultivos Tropicales. 37(2):102-107.

Domínguez, A.; Martínez, Y.; Pérez, Y.; Fuente, L.; Darias, R.; Sosa, M.; Rea, R. y Sosa, D. 2016. Comportamiento de variedades cubanas y venezolanas de frijol común, cultivados en condiciones de sequía. Revista Ciencia UNEMI. 9(20):68-75.

Estrada, W.; Jerez, E.; Nápoles, M. C.; Sosa, A.; Maceo, Y. C. y Cordoví, C. (2016). Respuesta de cultivares de frijol (Phaseolus vulgaris L.) a la sequía utilizando diferentes índices de selección. Cultivos Tropicales. 37(3):79-84.

FAO. 2018. FAOSTAT. Roma, Italia. http://www.fao.org/faostat/es/\#data/QC.

Faure, B.; Benítez, R.; León, N.; Chaveco, O. y Rodríguez, O. 2013. Guía técnica para el cultivo del frijol común (Phaseolus vulgaris L.). Cuba: ACTAF (Ed.). Agroecológica. 35 p.

Flores-Cruz, M. J.; García, A.; García, J. R.; Kohashi, J. y Ibarra, M. C. 2018. Diferencias fenológicas, morfológicas y de componentes del rendimiento entre una forma silvestre y domesticada de frijol común. Rev. Mex. Cienc. Agríc. 9(1):137-149.

Hernández, A.; Pérez, J. M.; Bosch, D. y Castro, N. 2015. Clasificación de los suelos de Cuba 2015. Mayabeque, Cuba. (Ed.). INCA. 93 p.

Hernández-Ochandía, D.; Rodríguez, M. G. y Holgado, R. 2018. Nematodos parásitos que afectan Phaseolus vulgaris L. en Latinoamérica y Cuba: especies, daños y tácticas evaluadas para su manejo. Rev. Protección Veg. 33(3):05-11.

Hernández-Ochandia, D.; Rodríguez, M. G.; Miranda, I.; Hernández, H. y Holgado R. 2016. Reacción de los genotipos BAT-306 y Triunfo-70 de Phaseolus vulgaris L. a Meloidogyne incognita (Kofoid y White) Chitwood. Rev. de Proteción Veg. 31(3):224-227.

Hernández-Ochandía, D.; Rodríguez, M. G.; Miranda, I.; Moreno, E.; Castro, I.; Peteira, B. y Holgado, R. 2018a. Reproducción y efecto nocivo de Meloidogyne incognita (Kofoid y White) Chitwood en Phaseolus vulgaris L. 'Cuba-Cueto-25-9'. Rev. Protección Veg. 33(2):05-10.

Hussey, R. S. and Barker, K. B. 1973. A comparison of methods for collecting inocula of Meloidogyne spp. including a new technique. Plant Disease Report. 57(3):1025-1028. 
Izquierdo, M.; Santana, Y.; García, A.; Carrodeguas, S.; Aguiar, I.; Ruiz, M.; Faure, B. y Monrabal, L. 2018. Respuesta agronómica de cinco cultivares de frijol común en un agroecosistema del municipio Consolación del Sur. Centro Agrícola. 45(3):11-16.

Maqueira, L. A.; Rojan, O.; Pérez, S. A. y Torres, W. 2017. Crecimiento y rendimiento de cultivares de frijol negro (Phaseolus vulgaris L.) en la localidad de Los Palacios. Cultivos Tropicales. 38(3):58-63.

Martínez, L.; Maqueira, L. A.; Nápoles, M. C. y Núñez, M. 2017. Efecto de bioestimulantes en el rendimiento de dos cultivares de frijol (Phaseolus vulgaris L.) biofertilizados. Cultivos Tropicales. 38(2):113-118.

Martínez, S. J.; Leiva, M.; Rodríguez, M.; Gómez, O.; Quintero, E.; Rodríguez, G.; García, A. y Cárdenas, M. 2015. Nuevas variedades de frijol común (Phaseolus vulgaris L.) para la Empresa Agropecuaria "Valle del Yabú", Santa Clara, Cuba. Centro Agrícola. 42(4):89-91.

Martinez, S. J.; Rodríguez, G.; Cárdenas, M.; García, O. y Colás, A. 2019. Respuesta morfofisiológica de cuatro cultivares de Phaseolus vulgaris en dos tipos de suelo. Centro Agrícola. 46(2):46-57.

MINAG. 2018. Lista oficial de variedades comerciales 32018. República de Cuba. 41 p.

Romero, C. S.; López, C.; Kohashi, J.; Miranda, S.; Aguilar, V. H. y Martínez, C. G. 2019. Cambios en el rendimiento y sus componentes en frijol bajo riego y sequía. Rev. Mex. Cienc. Agríc.10(2):351-364. 